\title{
The Evolution and Distance to SCORPIUS X-1
}

\author{
E. B. Fomalont
}

National Radio Astronomy Observatory, Charlottesville, VA, U.S.A.

\author{
C. F. Bradshaw \& B. J. Geldzahler \\ George Mason University, Fairfax, VA, U.S.A
}

\begin{abstract}
From three VLBA observations at $5 \mathrm{GHz}$, spanning 13 months, we have measured the trigonometric parallax of Sco X-1 of $0.23 \pm 0.28$ mas; hence its distance is $>1300 \mathrm{pc}$. This supports the hypothesis that the the $\mathrm{x}$-ray luminosity is near the Eddington Limit.

All three VLBA observations show a radio core of flux density $0.5 \mathrm{mJy}$ and size $<4$ mas. However, the third VLBA observation revealed two additional radio components, separated by 12 mas $(\approx 20 \mathrm{au})$ on opposite sides of the radio core. The evolution of these new components is unknown until additional observations can be made. The relative luminosity and separation of the two radio components are inconsistent with the Doppler beaming of two identical ejecta from the radio core.
\end{abstract}

Sco X-1 is one of about six low mass X-ray binary sources (LMXB) with quasiperiodic oscillations (QPO) of the $\mathrm{x}$-ray emission (e.g., Hasinger \& van der Klis 1989). The binary system is associated with a variable 13 -mag object, composed of a $1.5 M_{\odot}$ neutron star and a K-dwarf with a rotation period of 0.79 day (egs Cowley \& Crampton 1975). The radio emission is also variable on times scales of hours to days, with a range of $0.4 \mathrm{mJy}$ to $30 \mathrm{mJy}$. Previous radio observations have shown that Sco X-1 is less than 50 mas in size with a proper motion of 15 mas $\mathrm{yr}^{-1}$. Its distance is unknown (Fomalont \& Geldzahler 1991).

The VLBA observations at $5 \mathrm{GHz}$ were made in Aug 1995, Feb 1996 and Sep 1996. A small-diameter background radio source, $1^{\prime}$ NE of ScoX-1, was used as an in-beam calibrator. The 8-mJy NE source was successfully detected and imaged at all three epochs. Its structure did not change and we assume that it is stationary. Its calibration was applied to the Sco X-1 data which we then imaged and cleaned.

The images for Sco X-1 for the three epochs are shown in Figure 1. For the first two epochs, only a single component of flux density $0.5 \mathrm{mJy}$ was detected. The separation is consistent with the known proper motion. The contours show the image made from the third epoch. Three components are seen, but we believe that the faint, middle component is that associated with the single component of the previous two epochs. The fit to the parallax and proper motion are illustrated in Figure 2. Additional VLBA observations in 1997-1998 are planned to determine the evolution of the radio emission associated with Sco X-1.

Acknowledgments. The National Radio Astronomy Observatory is a facility of the NSF, operated under a cooperative agreement by Associated Universities, Inc.

\section{References}

Cowley, A. \& Crampton, D. 1975. ApJ, 201, L65-68.

Fomalont, E. B. \& Gelzahler, B. J. 1991. ApJ, 383, 289-294.

Hasinger, G. \& van der Klis, M. 1989, $A \& A, 225,79-96$. 


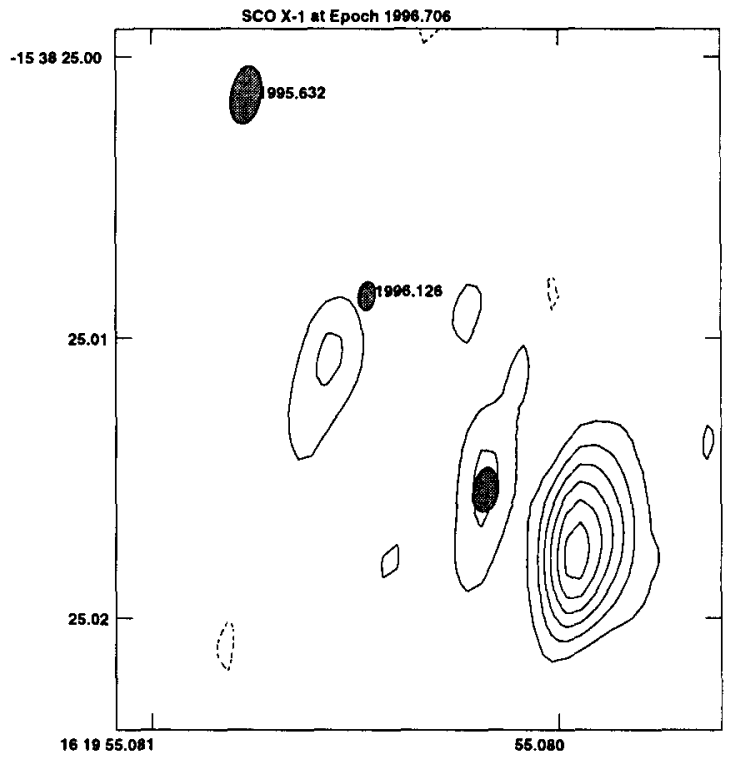

Figure 1. The Radio Structure of Sco X-1: The image for the three epochs are superimposed. The locations of the $0.5-\mathrm{mJy}$ radio component at epochs 1995.632 and 1996.126 are indication by the cross-hatched ellipses. The contour levels of $-0.2,0.2,0.4,0.6,0.8,1.0,1.2 \mathrm{mJy} /$ beam show the emission for epoch 1996.706. The cross-hatched ellipse centered in the weak middle component is identified with the radio component seen in the earlier two epochs. The coordinates are based on the position of the NE source which is assumed stationary in the sky.
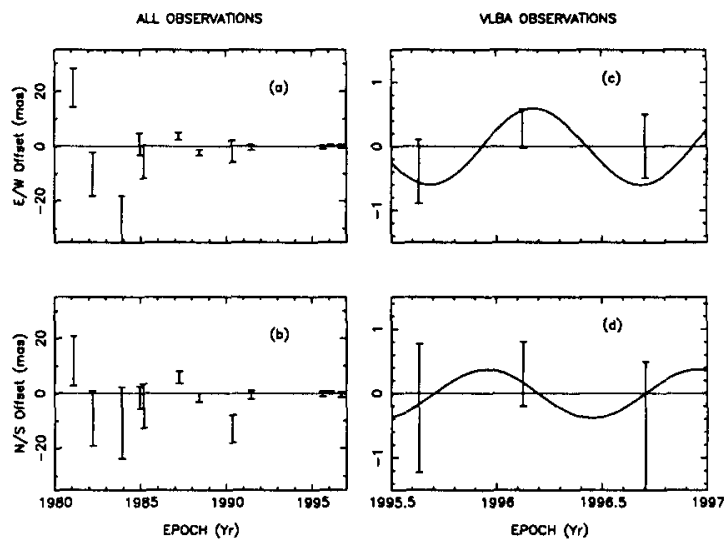

Figure 2. Kinematics of ScoX-1: The four panels show the residual motion of ScoX-1 with respect to the NE source after removing the best fit proper motion of $\mu_{x}=7.9 \pm 0.5, \mu_{y}=12.9 \pm 0.6 \mathrm{mas} \mathrm{yr}^{-1}$ and separation (1996.0) $x=34068.4 \pm 1.6, y=$ $60989.2 \pm 0.8$ mas. Panels (a) and (b) show the consistency of the fit, from the early VLA observations to the most recent VLBA observations. Panels (c) and (d) show the three VLBA results, suitably magnified. The line at zero shows the expected residual for no parallax $\pi=0$ and the sinusoid for $\pi=0.60$ mas. 